\title{
Les motivations et les moyens de quelques instrumentations " in situ » d'ouvrages en mer
}

\author{
The underlying reasons and the means for some in-situ \\ instrumentation used for works at sea
}

\author{
M. Olagnon, M. Prevosto, Ph. Ozanne
}

IFREMER

Le rapport donne quelques exemples d'instrumentations réalisées ou prévues sur un certain nombre de plate-formes fixes, en vue d'obtenir des éléments de réponses aux questions que se posent les responsables de l'industrie pétrolière concernant les techniques de conception de ces plate-formes : validité des calculs, garantie d'un comportement normal des structures jusqu'à la fin de la durée de vie prévue, détection des endommagements graves, possibilité de prolongation de la durée de vie des structures au-delà de la durée initialement prévue.

This report gives some examples of instrumentation used or anticipated for use on some fixed platforms, aimed at obtaining answers to the questions posed by the heads of petroleum industries concerning the design techniques of these platforms: validity of calculations, guarantee of a normal behaviour of structures until the end of their anticipated serviceable lifetime, detection of serious damage, possibility of extending the serviceable lifetime of structures beyond the lifetime initially anticipated.

\section{Le contexte}

La production pétrolière offshore a connu un très vaste développement dans les années 70, quand, stimulés par la crise et la hausse des prix du brut, les opérateurs ont dû se tourner vers de nouvelles régions d'exploration et de production. Les techniques de conception et de construction de plateformes fixes ont été poussées à leurs limites pour relever un défi technologique qui était encore de la science-fiction dans la décennie précédente. Depuis lors, les responsables de l'industrie pétrolière n'ont cessé de se poser deux questions :

1. Les hypothèses et les outils utilisés pour le calcul sont-ils et étaient-ils satisfaisants ?

2. Jusqu'à quel point peut-on garantir un comportement normal pour une structure jusqu'à la fin de sa durée de vie prévue, et dans l'éventualité d'endommagements graves, comment les détecter à temps pour éviter d'avoir à interrompre la production, ou pire, une ruine complète de la plateforme, et inversement pour éviter de coûteuses réparations pour des dommages anodins?

Une troisième interrogation est apparue récemment, liée aux découvertes nouvelles et à la chute des cours :

3. La durée de vie des structures peut-elle être prolongée au-delà de ce qui était initialement prévu ?

L'examen des causes d'incidents et d'accidents survenus jusqu'à présent a permis de répondre partiellement à la première question en décernant une mention honorable aux techniques de conception, au moins en ce qui concerne les concepts classiques (par opposition aux structures souples, d'apparition récente). Par contre, les questions 2 et 3 deviennent de plus en plus cruciales et n'ont pas reçu à ce jour de réponse satisfaisante. De telles réponses ne peuvent résulter, à notre avis, que d'efforts d'interprétation d'expériences en vraie grandeur et de l'amélioration subséquente des modèles numériques et des méthodes de calcul d'une part, et d'autre part de l'élaboration de techniques de suivi du comportement à la fois efficaces et bon marché. 


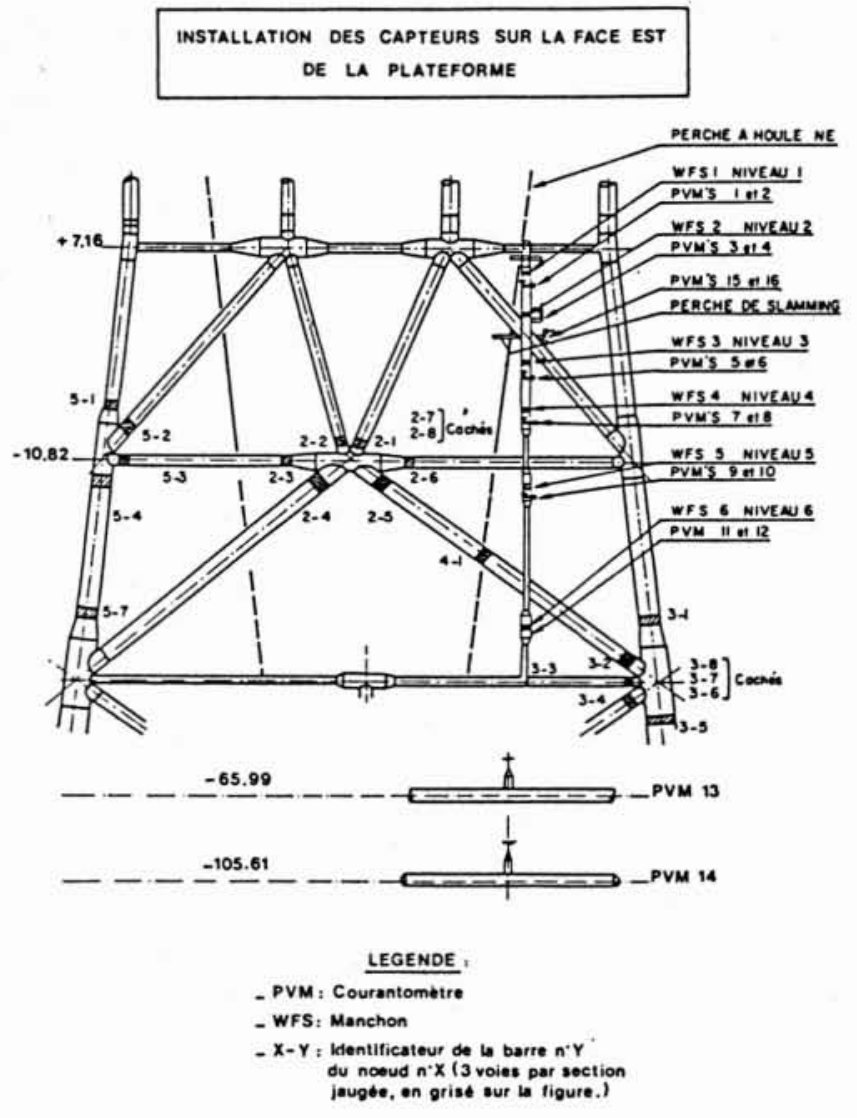

Figure 1. - Implantation des capteurs sur le jacket de Forties Bravo.
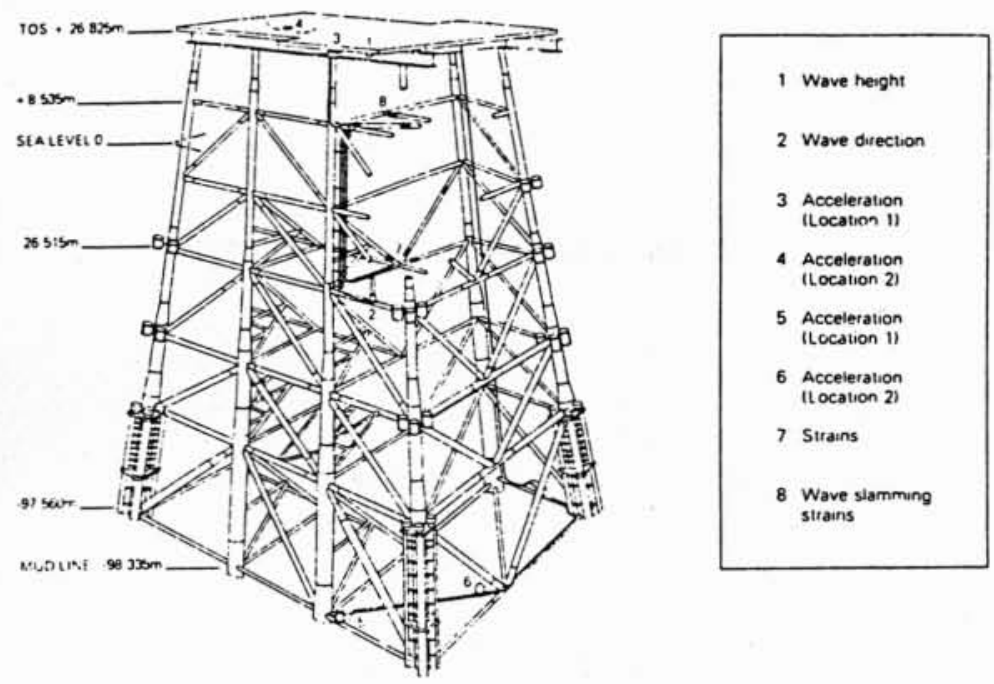

Figure 3. - Le jacket de DP2 (champ de FRIGGS). (D'après OTC $3997-1980$ ).

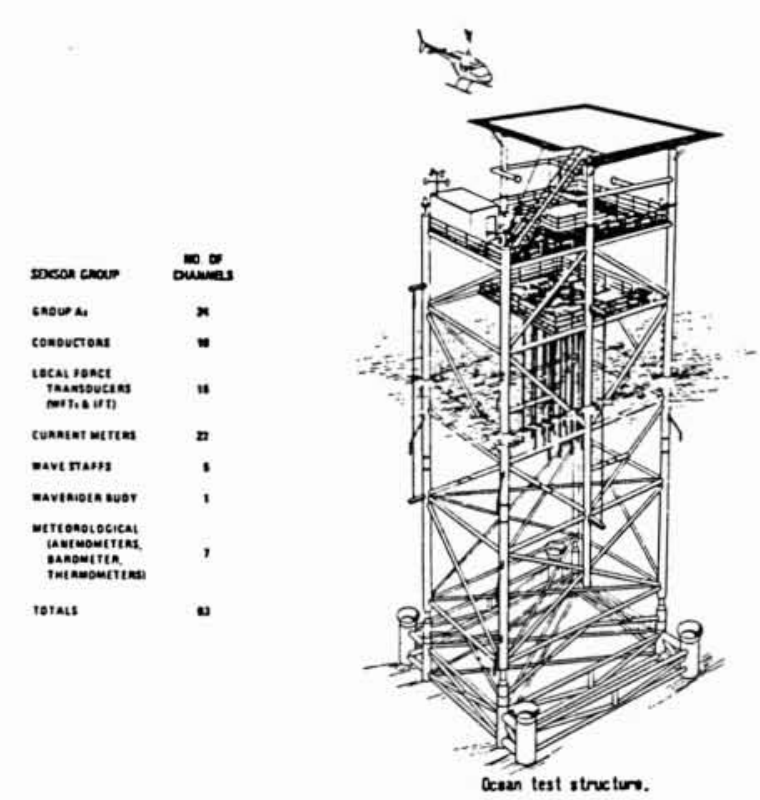

Figure 2. - (D'après EUROPEC, 1978).

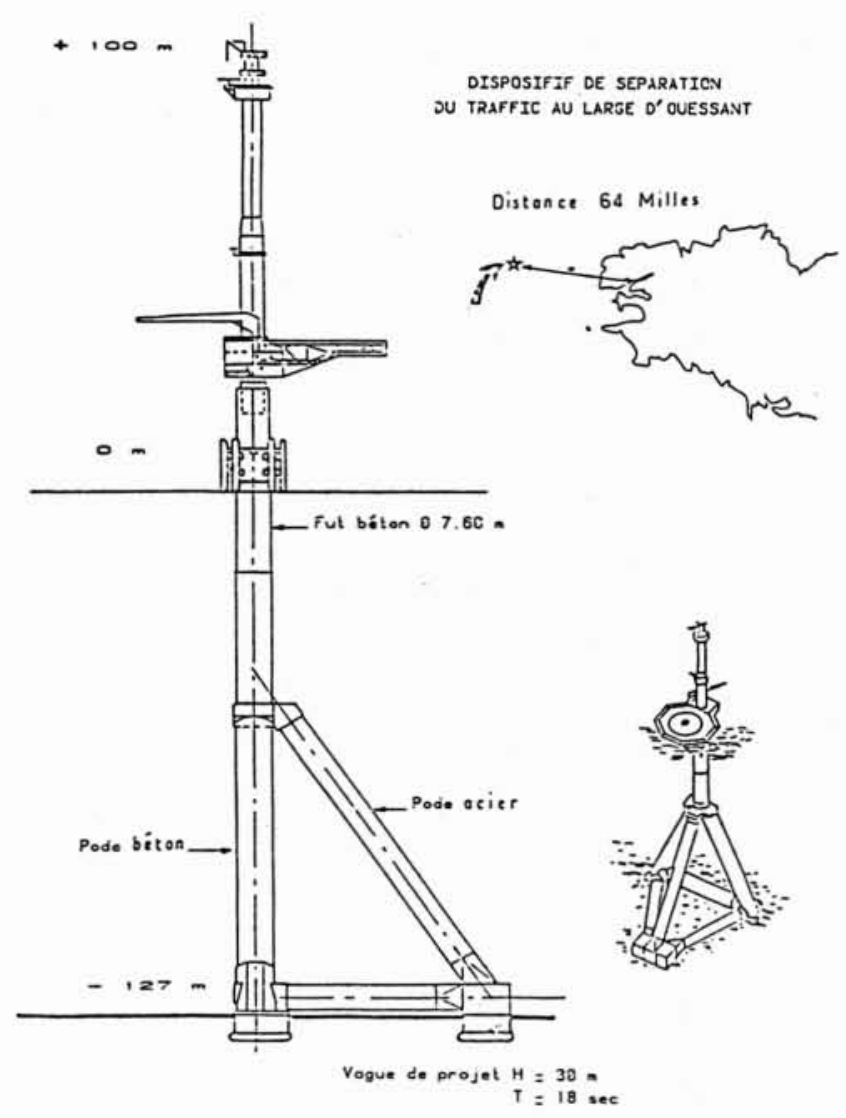

Figure 4. 


\section{Quelques expériences « in situ »}

Conscients des risques qu'ils prenaient, opérateurs et organismes de certification ont conduit des campagnes d'instrumentation "in situ ", souvent de grande ampleur, visant principalement à répondre à la première question : la validation de la conception par une meilleure compréhension des phénomènes d'interaction houle-structure. II n'entre pas dans notre dessein d'effectuer une revue exhaustive des différentes expériences " in situ " qui ont pu être faites, mais d'illustrer par quelques exemples les caractéristiques de ces instrumentations.

\section{Forties Bravo Structural Performance Monitoring Pro- gram (British Petroleum)}

Cette campagne, qui a duré de 1976 à 1978, a été l'une des premières de grande ampleur en Mer du Nord. D'un coût élevé (près d'un million de livres sterling de l'époque), cette expérience était principalement axée sur des mesures de forces et de contraintes et de leur corrélation avec l'environnement : houle et courants. Ceci peut être vérifié sur la figure 1 qui montre les différents capteurs utilisés sur la plateforme : courantomètres, manchons de mesure d'efforts, jauges de contraintes.

\section{Ocean Test Structure (EXXON)}

Cette expérience, menée en 77-78, présente la particularité d'avoir utilisé une plateforme totalement expérimentale (sans équipement pétrolier de production) et de faible taille par rapport aux plateformes de Mer du Nord $\left(1 / 5^{\circ}\right)$ telles que Forties Bravo ou Frigg DP2. Ses objectifs étaient similaires à ceux du Programme Forties, servis par une instrumentation mieux conçue du fait de l'absence de contraintes d'exploitation.

\section{Frigg DP2 (ELF)}

L'instrumentation installée sur cette plateforme fonctionne de manière permanente depuis fin 78 . Elle est beaucoup moins spécifique que les précédentes de l'interaction houle-structure et permet, grâce à la présence de quatre accéléromètres bi-directionnels, le recalage de modèles éléments finis ainsi que le suivi des propriétés du comportement dynamique de la structure.

\section{Résultats obtenus à partir de ces instrumentations}

Un certain nombre de facteurs ont rendu très décevants les résultats des premières campagnes d'instrumentation offshore, principalement :

- la mauvaise qualité des données consécutive à l'hostilité du milieu dans lequel elles furent acquises;

- l'absence de préparation de la communauté technologique et scientifique qui ne disposait à l'époque ni de méthodes ni d'outils adaptés au traitement et à l'interprétation des données recueillies;

- le coût beaucoup plus élevé que prévu de l'acquisition des mesures, qui a amputé d'autant le budget prévu pour l'interprétation;

- et probablement les trop grands espoirs qui avaient été mis dans ces campagnes en visant de grandes améliorations des méthodes et hypothèses de calcul, qui n'ont pas été possibles.

Ainsi qu'il a déjà été dit plus haut, ces méthodes de calcul se sont révélées honorables, et l'utilisation des expérimentations " in situ " pour des validations et améliorations, au niveau des formules de modélisation de l'action de la houle à prendre en compte pour la conception, ne se justifie que pour des concepts nouveaux.

L'utilisation des mesures pour le recalage des modèles éléments-finis, la surveillance de l'intégrité structurelle et le suivi à long terme du comportement ont nécessité de longs développements dont certains sont encore en cours, entre autres à l'IFREMER.

Des résultats probants ont déjà été obtenus sur chacun de ces points, et ce grâce à des méthodes originales de traitement du signal qui permettent une évaluation extrêmement précise du comportement dynamique des structures. Cette capacité nouvelle à tirer un excellent parti des expérimentations "in situ", jointe à la faible confiance qui peut être accordée à la généralisation à la vraie grandeur d'essais en laboratoire pour des structures compliquées, permet d'envisager de nouvelles campagnes, visant soit la surveillance de l'intégrité structurelle, soit la validation de modèles de comportement, soit même une amélioration des paramètres de la conception, en particulier en ce qui concerne les propriétés du sol, les conditions inhabituelles en termes de nombres adimensionnels caractérisant l'écoulement, ou les méthodes nouvelles de calcul.

\section{Des instrumentations à venir}

\section{Heimdal (ELF)}

L'instrumentation d'Heimdal a été spécialement conçue en vue d'opérer une surveillance de la structure par des mesures régulières de sa rigidité, et, dans le cas d'une variation constatée, de préciser au mieux la nature et la localisation probables de sa cause. Elle consiste en des mesures d'accélérométries effectuées au sommet des piles et à des niveaux variables par un ou deux boîtiers mobiles descendus dans des tubes-guides solidaires de celles-ci. Un logiciel spécialisé, MASI, développé en collaboration par l'IFREMER et l'Institut norvégien SINTEF, permettra de tirer le meilleur parti possible des mesures, dont les premières seront effectuées à l'automne 86 .

\section{Pitot}

La mise en place, prévue pour l'été 1988, de l'Aide majeure à la navigation, un phare de cent mètres de haut par $120 \mathrm{~m}$ de fond destiné à la séparation du trafic maritime au large d'Ouessant, avait permis de mettre sur pied un vaste programme d'instrumentation, dont les objectifs étaient :

- l'amélioration de la connaissance et de la prédiction des propriétés mécaniques du sol devant supporter la structure; 
- la vérification des formulations des forces de houle pour des valeurs inhabituelles des nombres de Reynolds et de Keulegan-Carpenter;

- la validation du calcul dynamique de la structure et la surveillance en service de son intégrité;
- la mesure du comportement du nœud principal mixte béton-acier et de la fatigue aux points les plus chargés; - la vérification de l'efficacité du système de protection cathodique, des méthodes qui ont permis de le concevoir et le suivi dans le temps des problèmes de corrosion.

\section{Conclusions}

Ainsi qu'on peut le voir sur ces derniers exemples, les instrumentations en vraie grandeur sont des facteurs déterminants pour apporter des éléments de réponse aux trois questions posées plus haut. Les grands axes qui les dirigent sont les suivants :

- instrumenter les structures de conception originale et nouvelle (plateformes à lignes tendues, structures souples, etc.);

- améliorer la connaissance des paramètres encore heuristiques de la conception (propriétés mécaniques du sol,...);

- suivre à peu de frais le comportement de certaines structures dont on souhaite pouvoir réévaluer ou prolonger la durée de vie.

Contrairement à ce qui s'est produit pour les premières campagnes d'instrumentation, les ingénieurs disposent maintenant d'outils performants pour déterminer et extraire les informations pertinentes dans la masse énorme de données que produit une instrumentation "in situ ". Les opérateurs pétroliers peuvent donc concevoir en connaissance de cause des instrumentations avec d'excellentes chances d'atteindre les objectifs précis qu'ils se seront fixés. Il appartient maintenant à ceux qui ont développé et exploitent ces outils de les mettre à la disposition des autres secteurs industriels auxquels ils peuvent être utiles. 